\title{
Uniportal video-assisted thoracoscopy approach to the management of non-pulmonary diseases of the chest
}

\author{
Ching Yeung ${ }^{1,2}$, Jennifer Dawson $^{1}$, Sebastien Gilbert ${ }^{1,2}$ \\ ${ }^{1}$ The Ottawa Hospital Research Institute, ${ }^{2}$ Faculty of Medicine, Department of Surgery, University of Ottawa, The Ottawa Hospital - General \\ Campus, Ottawa, ON, Canada \\ Contributions: (I) Conception and design: S Gilbert, C Yeung; (II) Administrative support: None; (III) Provision of study materials or patients: None; \\ (IV) Collection and assembly of data: None; (V) Manuscript writing: All authors; (VI) Final approval of manuscript: All authors. \\ Correspondence to: Sebastian Gilbert, MD. Division of Thoracic Surgery, The Ottawa Hospital - General Campus Suite 6363, 501 Smyth Road, \\ Ottawa, ON K1H 8L6, Canada. Email: sgilbert@toh.on.ca.
}

\begin{abstract}
The use of uniportal video-assisted thoracoscopy (u-VATS) is becoming a commonly used surgical technique and can be an effective approach for the surgical treatment of many pulmonary and nonpulmonary conditions. This review article summarizes current medical evidence informing the practice of u-VATS for treating non-pulmonary conditions including hyperhidrosis, hemothorax, pleural effusion, and thymic disease.
\end{abstract}

Keywords: Uniportal video-assisted thoracoscopy (u-VATS); hyperhidrosis; thymectomy; hemothorax; pericardial effusion; empyema

Submitted Jan 26, 2019. Accepted for publication Mar 07, 2019.

doi: $10.21037 /$ jtd.2019.03.21

View this article at: http://dx.doi.org/10.21037/jtd.2019.03.21

\section{Introduction}

The use of uniportal video-assisted thoracoscopy (u-VATS) to diagnose and treat non-pulmonary conditions has been utilized since the early 2000s with early work in sympathectomy (1-3). The publication of u-VATS wedge resection in 2004 stimulated a wider interest in the use of $\mathrm{u}$-VATS (4). Since then, the acceptance of the approach has varied worldwide with greater enthusiasm in Europe and Asian and nascent interest in North America (5). In this article, we highlight the indications for u-VATS in the management of hyperhidrosis, the management of effusions and collections in the thoracic cavity, and the treatment of thymic disease.

U-VATS can be performed using various approaches including intercostal, subxiphoid, transcervical, and transaxillary (6). This article will focus on intercostal u-VATS and will describe the use of subxiphoid and transcervical u-VATS approaches for specific conditions. Briefly, when performing intercostal u-VATS, patients are placed in lateral decubitus, similar patient position to multi-port VATS (m-VATS). Under general anesthesia and single-lung ventilation, a $3-5 \mathrm{~cm}$ access incision is required between mid and anterior axillary lines in the $5^{\text {th }}$ intercostal space. A $30^{\circ}$ scope, positioned in the posterior portion of the incision, is then used to minimize potential conflict between the scope and the surgical instruments. Surgical instruments that can be used for u-VATS include instruments specific to VATS, laparoscopic instruments, and open surgical instruments $(7,8)$.

For a subxiphoid u-VATS approach, the patient is placed in a supine position. A vertical incision is fashioned over the xiphoid and the xiphoid process is resected if required. The xiphoid is separated from the rectus abdominus to allow a finger to bluntly dissect the posterior sternal table away from the anterior mediastinum. A single incision laparoscopic surgery (SILS) port is inserted, and a $30^{\circ}$ scope is introduced. The anterior mediastinum is dissected open under the sternum (9-11).

\section{Primary palmar and axillary hyperhidrosis}

Some of the earliest applications of u-VATS have been for 
Table 1 Post-operative sympathectomy complications reported in $\mathrm{m}$-VATS and u-VATS

\begin{tabular}{lcc}
\hline Complication & M-VATS (\%) & U-VATS (\%) \\
\hline Compensatory hyperhidrosis & $10-93$ & $21-98$ \\
Pneumothorax & $0-2.4$ & $0.5-10$ \\
Horner's syndrome & $0-0.5$ & $0-7$ \\
\hline
\end{tabular}

m-VATS, multiport video-assisted thoracoscopy; u-VATS, uniportal video-assisted thoracoscopy.

non-pulmonary conditions including sympathectomy for primary palmar and axillary hyperhidrosis. The Society of Thoracic Surgeons (STS) recommends a surgical approach for palmar and palmar-axillary primary focal hyperhidrosis as it is the most effective treatment for these conditions (12). Large centre experiences with u-VATS for treating palmar and axillary hyperhidrosis have reported operating times under 30 minutes and success rates over $90 \%(2,3)$. U-VATS and m-VATS case series studies have reported the complication rates for compensatory hyperhidrosis, pneumothorax, and Horner's syndrome (Table 1) (2,3,13-18). Other complications include gustatory hyperhidrosis and bradycardia $(12,19)$. The rate of recurrence for patients with hyperhidrosis who have underwent a u-VATS sympathectomy has been reported as $17 \%$ recurrence at 5 years postprocedure (20). A study directly comparing u-VATS to $\mathrm{m}$-VATS has yet to be conducted. The risk of compensatory hyperhidrosis should be discussed with patients in the preoperative setting as this can greatly affect quality of life.

\section{Technique}

Patients are placed in a semi-sitting or supine position and a $1-2 \mathrm{~cm}$ incision is fashioned along the $3^{\text {rd }}$ intercostal space. The level of sympathetic chain of interest is identified and transected, along with the accessory nerve of Kuntz around the sympathetic ganglia $(2,3)$. For palmar hyperhidrosis, disruption of the sympathetic ganglia at $\mathrm{T} 3$ has been suggested to be the most effective approach for alleviating excessive sweating. Sympathectomy at the T4 level may also be considered as it has a lower incidence of compensatory hyperhidrosis compared to the T3 approach (12). For axillary hyperhidrosis, a $\mathrm{T} 5$ transection is recommended. If palmar-axillary hyperhidrosis is present, transection of the sympathetic ganglia should occur at T4 and T5. Sympathetic chain disruption can be conducted through electrocautery, scissors, or clipping. A chest tube should be placed at the end of the procedure $(2,3,12,21)$.

\section{Empyema, pleural and pericardial effusions}

U-VATS has been used in the diagnosis and management of thoracic cavity effusions.

\section{Empyema}

Most cases of empyema can be successfully treated with tube thoracoscopy drainage and fibrinolytics (22). However, for stage II and stage III empyema, the European Association for Cardio-Thoracic Surgery supports the role of VATS as a treatment approach. Evidence suggests that the VATS approach may result in a shorter hospital stay, reduced morbidity, and reduced mortality $(23,24)$. The uniportal literature on empyema is limited. In a retrospective study, 30 patients who underwent u-VATS decortication were compared to 34 patients who underwent open decortication for stage II-III empyema. U-VATS was associated with statistically significant lower blood loss, 4-day shorter hospital stay, and lower complication rates $(10 \%$ vs. $16 \%$, $\mathrm{P}<0.001)(25)$. There were 3 conversions from uniportal to m-VATS and no conversions to an open approach. Limitations of this study include a lack of inclusion criteria for the open group. More comparative evidence on m-VATS and $\mathrm{u}$-VATS for the management of empyema is required.

\section{Technique}

A pre-operative ultrasound and computed tomography (CT) scan is suggested to determine the stage of empyema and to assist with pre-operative planning prior to u-VATS. An incision located at the level of the $7^{\text {th }}$ or $8^{\text {th }}$ intercostal space is recommended to facilitate access to the most dependent portions of the chest cavity. During evacuation of the fluid, samples are obtained for culture and sensitivity. Surgical instruments such as Yankauer suction, curved ring forceps, and cotton swabs in forceps, are used to release adhesions and drain loculation. The parietal pleural cortex is dissected free from the diaphragm and chest wall, and from the thickened visceral pleura from the underlying lung parenchyma $(24,25)$.

\section{Pleural effusion of unknown etiology}

The presence of pleural effusions can be the result of many different medical conditions including congestive heart failure, pneumonia, and malignancy. Prompt investigation is important for proper diagnosis and appropriate treatment. Surgery should be considered if cytology from percutaneous 
drainage samples is non-diagnostic or biopsy of pleural or parenchymal lesions is indicated $(9,26)$. A subxiphoid u-VATS may be ideal for certain patients with undiagnosed pleural effusion. For example, if bilateral effusions are present, subxiphoid u-VATS allows access to both sides of the thorax (9). A subxiphoid u-VATS may also be used in patients who are too ill to tolerate lateral decubitus position due to a large effusion.

In a case series of 329 patients undergoing intercostal u-VATS for diagnosis and management of pleural effusion, a diagnosis was secured in $100 \%$ of the cases (malignant 281/329; benign 48/329) (26). Of note, 4 patients (1\%) reported in this series required intensive care unit (ICU) admission and died within 30 days of the procedure. The cause of death was not stipulated in the article. Other morbidity included atrial fibrillation (1\%), prolonged air leak (4\%), and persistent drainage requiring talc pleurodesis (5\%) (26).

\section{Technique}

For intercostal u-VATS, imaging is used to select the optimal intercostal space to access the pleural fluid. The incision is generally along the midaxillary line. The camera is inserted under direct visualization. Pleural fluid can be processed for cytology, and microbial cultures. Any suspicious nodules visualized can be biopsied or resected for pathologic analysis. If malignancy is confirmed by analysis of frozen sections, talc pleurodesis can be performed during the same operation (26). For subxiphoid u-VATS, access is obtained as described earlier in this article. The pleura is grasped with forceps and incised. Pleural fluid can then be drained (9). A chest tube is secured through the incision site at the end of the procedure.

\section{Pericardial effusion}

An approach that includes the creation of a pericardial window can be used to relieve a symptomatic patient experiencing tamponade from a malignant pericardial effusion and for patients who require serial pericardiocentesis. In u-VATS, both the intercostal and subxiphoid approaches have been used for the management of pericardial effusion. Evidence in the literature specific to the use of u-VATS was limited to case series reports at the time of this review $(27,28)$. Reported morbidities include pulmonary air leak from inadvertent injury to lung $(0-11 \%)$; arrhythmia (0-13\%); atelectasis (0-19\%). There was no perioperative mortality reported. Recurrence was less than
$5 \%$ at $6-7$ months following surgery $(27,28)$.-

\section{Technique}

For intercostal u-VATS pericardial drainage, patients are placed in a supine or semi-lateral decubitus position. An incision is fashioned in the $5^{\text {th }}$ intercostal space over the anterior axillary line. We acknowledge that a rightsided approach may provide more working space and that some surgeons may select the operative side based on the location of greatest fluid accumulation as determined by preoperative imaging. However, we recommend a left-sided approach so that the pericardial incision can be placed over the left ventricle, thereby decreasing the risk of potentially lethal cardiac laceration especially in the presence of intrapericardial adhesions. Dissection is carried out anterior to the phrenic nerve and a portion of the pericardial is resected to create a window. The pericardium is sent for pathologic analysis. In the absence of any intrapericardial adhesions, a suction cannula may be used to aspirate the fluid. If indicated, instillation of a sclerosing or cytotoxic agent can be conducted during the same procedure (27). A subxiphoid u-VATS has been used as an alternative approach to drain the pericardium (29). However, in a non-trauma setting, our preferred approach would be a left sided intercostal approach. The reasoning is because in a non-traumatic setting, there may be the presence of adhesions and loculations. A subxiphoid approach would place the pericardial incision anteriorly in the vicinity of the right ventricle which is very thin walled and susceptible to injury from avulsion of intra-pericardial adhesions. If the subxiphoid u-VATS approach is the only available option, then every effort should be made to drain the pericardium through its left-sided or diaphragmatic surface.

\section{Hemothorax}

Hemothoraces can be caused by trauma, iatrogenic injury, or an infection such as aspergilloma. Indications for surgical interventions to treat a hemothorax include the presence of persistent bleeding and a retained hemothorax $(6,30)$. There are differing opinions related to the timing of surgical interventions in the treatment of the patient with a hemothorax. There are several advantages related to early surgical intervention. First, it reduces the risk of activating local fibrinolysis thereby reducing the risk that a person may experience a recurrence of their hemothorax. Second, unnecessary transfusions may be avoided by earlier control of the source of bleeding. Third, the placement of chest 
tubes is under direct visualization for optimal pleural space drainage (31). However, routine early intervention, may increase the incidence of unnecessary surgical procedures in cases where insertion of a chest tube would have sufficed. Conversely, delaying surgical intervention may result in empyema and may also increase the risk of conversion to open thoracotomy at the time of attempted drainage $(6,31)$.

The effectiveness of minimally invasive approaches for treating hemothoraces has not been widely investigated. In one case series, 12 patients with active bleeding underwent $\mathrm{u}$-VATS (32). Inclusion criteria for surgical management included chest-tube output over $250-500 \mathrm{~mL}$ of blood in the first 1-3 hours in an otherwise hemodynamically stable patient. Investigators reported no intra-operative conversions and 1 (8\%) postoperative pneumothorax. Mortality was $17 \%(2 / 12)$ and was related to patient co-morbidities. The mean hospital stay was 11 days. The rebleeding rate and need for re-operation were not reported.

\section{Technique}

To treat a hemothorax, a uniportal incision is made in the $4-6^{\text {th }}$ intercostal space. The source of the active bleed should first be identified and managed. A Yankauer suction tip is used to evacuate the blood. A large bore laparoscopic suction-irrigator is used to apply copious amounts of irrigation. Endoscopic graspers or ring forceps can be used to remove clots. In the case of a retained hemothorax, removal of the clot and decortication of lung will result in a successful procedure. For penetrating injuries, the lung parenchyma and chest wall should be examined to determine the source of the bleed (6). The advantage of u-VATS is that the incision can rapidly be extended to a mini-thoracotomy or larger thoracotomy if necessary. The decision to convert will depend on the training and experience of the surgeon and the clinical situation.

\section{Thymic diseases}

Surgery is indicated in the management of nonthymomatous myasthenia gravis, thymic tumours, and undetermined lesions of the anterior mediastinum, not thought to be lymphoma. A randomized controlled trial involving 126 patients with nonthymomatous myasthenia gravis compared management of transsternal thymectomy with prednisone versus prednisone alone. Results indicated that patients who underwent thymectomy with prednisone had lower prednisone requirements (44vs. $60 \mathrm{mg}$ ), reduction in myasthenia gravis score (6.15 vs. 8.99) and exacerbations at
3 years compared to those who did not undergo surgery (33).

Patients who underwent a complete resection for thymoma had significantly better 10-year survival (71-76\%) compared to those who underwent an incomplete resection $(9-28 \%)(34,35)$. Overall recurrence is $9-15 \%$ and recurred on average 5 years after complete resection $(34,35)$.

Traditionally, transsternal thymectomy was the standard approach as it permitted complete resection of the thymic gland and removal of ectopic thymic tissue in myasthenia gravis patients. However, as experience with minimally invasive techniques increased, VATS thymectomy has been used in nonthymomatous myasthenia gravis and thymomas (36). M-VATS thymectomy results in less blood loss, shorter chest tube duration, less pain, and shorter hospital stays when compared to transsternal thymectomy (36). Results from two systematic reviews indicate that minimally invasive versus open thymectomy in selected patients had similar oncological outcomes and disease-free survival $(37,38)$.

To minimize the risk of tumour seeding, indications for minimally invasive thymectomy include patients with nonthymomatous myasthenia gravis, and well encapsulated thymoma $<4 \mathrm{~cm}(39,40)$. Tumor larger than $4 \mathrm{~cm}$ and suspicion of innominate vein invasion were considered contra-indication to u-VATS thymectomy.

A surgical approach from the ipsilateral side of the tumor is suggested as it permits dissection under direct visualization. If the tumor is midline, the approach may be right or left-sided. Although the right-sided approach provides more working space, visualization of the left phrenic nerve and the proximal innominate vein is suboptimal. This problem can be overcome in m-VATS by adding a contra-lateral port. This option would obviously convert a u-VATS approach to m-VATS.

Subxiphoid and transcervical u-VATS present a distinct advantage in that the pain associated with intercostal incisions can be avoided altogether $(10,41)$. In subxiphoid u-VATS, the camera is midline, allowing for good visualization of both superior poles of the thymus and both phrenic nerves (10). Regardless of the approach selected, the tumor should ideally be dissected last as part of a "notouch" technique (39). There have been a few reported case series of u-VATS thymectomy reporting comparable outcomes to m-VATS thymectomy $(8,39,42)$.

\section{Technique}

When performing an intercostal u-VATS, the patient is placed in a supine or semi-supine and a roll is placed 
longitudinally, parallel to the spine. An incision is usually made in the $4^{\text {th }}$ or $5^{\text {th }}$ intercostal space. $\mathrm{CO}_{2}$ insufflation is obtained to low pressure of $8 \mathrm{mmHg}$. The camera is directed at the cardiophrenic angle. The mediastinal fat anterior to the phrenic nerve is grasped and dissected free from the phrenic nerve. The dissection proceeds in a caudal to cranial from the cardiophrenic angle, continuing along the anterior border of the phrenic nerve, towards the innominate vein. Opening of the contralateral pleura allows for improved visualization of the contralateral phrenic nerve. The upper poles are dissected cranially to the origin of the thyrothymic ligament, and the thymus is retracted laterally to allow ligation of thymic venous tributaries. All adipose tissue in the anterior mediastinum between both phrenic nerves is resected en bloc with the thymus gland. In myasthenic patients, adipose tissue in the aortopulmonary window is also resected. The specimen is placed in a retrieval bag prior to removal $(39,42)$.

For subxiphoid u-VATS, the surgical principles and steps are the same. Access is described earlier in the paper. A SILS port is used and a $30^{\circ}$ camera is inserted and $\mathrm{CO}_{2}$ insufflation is obtained $(10,11)$.

In a transcervical u-VATS thymectomy, the patient should be placed supine with the neck hyperextended. A collar incision, one finger breadth above the sternal notch is required. The infrahyoid (strap) muscles are divided. A sternal retractor is placed to lift the sternum and a $0^{\circ}$ or $30^{\circ}$ camera is inserted. The thymic gland is dissected from the pleura and pericardium using blunt and sharp dissection. If intra-operatively there are concerns about an incomplete resection, a u-VATS left incision can be fashioned to assist with a more radical excision (41).

\section{Conclusions}

Surgeon's comfort with u-VATS has increased as more research is published on the favourable outcomes of u-VATS for the patient. In the lobectomy literature, a meta-analysis favoured u-VATS over m-VATS for length of stay, shorter duration of chest tube drainage, and less morbidity (43). An additional benefit of u-VATS is the ergonomic advantages that it provides the surgeon. In m-VATS, the surgeon is often standing sideways and must rotate the torso or lean to operate. They must also turn the head to view the monitor screen. In u-VATS the viewing direction is realigned with the working direction allowing for better ergonomics. There is less head, neck and back rotation and therefore less potential for physical strain (44).
Although the evidence in support of u-VATS remains limited, it is emerging that u-VATS may be used to provide safe surgical management for conditions, such as, hyperhidrosis, pleural and pericardial fluid accumulation, and thymic disease. As we continue to strive to decrease morbidity and shorten postoperative hospitalization, and with the development of new single-port access technological platforms, surgeons should consider integrating u-VATS as part of their armamentarium in the treatment of intra-thoracic disease.

\section{Acknowledgments}

None.

\section{Footnote}

Conflicts of Interest: The authors have no conflicts of interest to declare.

\section{References}

1. Migliore M. Efficacy and safety of single-trocar technique for minimally invasive surgery of the chest in the treatment of noncomplex pleural disease. J Thorac Cardiovasc Surg 2003;126:1618-23

2. Lin TS, Kuo SJ, Chou MC. Uniportal endoscopic thoracic sympathectomy for treatment of palmar and axillary hyperhidrosis: analysis of 2000 cases. Neurosurgery 2002;51:S84-7.

3. Lai YT, Yang LH, Chio CC, et al. Complications in patients with palmar hyperhidrosis treated with transthoracic endoscopic sympathectomy. Neurosurgery 1997;41:110-3.

4. Rocco G, Martin-Ucar A, Passera E. Uniportal VATS wedge pulmonary resections. Ann Thorac Surg 2004;77:726-8.

5. Yeung $\mathrm{C}$, Gilbert $\mathrm{S}$. The state of uniportal video-assisted thoracoscopic surgery in North America: a survey of thoracic surgeons. J Vis Surg 2018;4:19.

6. Sanna S, Bertolaccini L, Brandolini J, et al. Uniportal video-assisted thoracoscopic surgery in hemothorax. J Vis Surg 2017;3:126.

7. French DG, Thompson C, Gilbert S. Transition from multiple port to single port video-assisted thoracoscopic anatomic pulmonary resection: early experience and comparison of perioperative outcomes. Ann Cardiothorac Surg 2016;5:92-9. 
8. Gonzalez-Rivas D. Uniportal thoracoscopic surgery: from medical thoracoscopy to non-intubated uniportal videoassisted major pulmonary resections. Ann Cardiothorac Surg 2016;5:85-91.

9. Martínez-Téllez E, Trujillo-Reyes JC, et al. Subxiphoid video-thoracoscopy. J Thorac Dis 2018;10:S2643-8.

10. Suda T. Subxiphoid thymectomy: single-port, dual-port, and robot-assisted. J Vis Surg 2017;3:75.

11. Zieliński M, Rybak M, Solarcyzk-Bombik K, et al. Subxiphoid uniportal VATS thymectomy. J Vis Surg 2017;3:171-5.

12. Cerfolio RJ, De Campos JR, Bryant AS, et al. The Society of Thoracic Surgeons expert consensus for the surgical treatment of hyperhidrosis. Ann Thorac Surg 2011;91:1642-8.

13. Menna C, Ibrahim M, Andreetti C, et al. Long term compensatory sweating results after sympathectomy for palmar and axillary hyperhidrosis. Ann Cardiothorac Surg 2016;5:26-32.

14. Kwong KF, Cooper LB, Bennett LA, et al. Clinical experience in 397 consecutive thoracoscopic sympathectomies. Ann Thorac Surg 2005;80:1063-6.

15. Findikcioglu A, Kilic D, Hatipoglu A. Is clipping superior to cauterization in the treatment of palmar hyperhidrosis? Thorac Cardiovasc Surg 2014;62:445-9.

16. de Campos JR, Kauffman P, Werebe Ede C, et al. Quality of life, before and after thoracic sympathectomy: report on 378 operated patients. Ann Thorac Surg 2003;76:886-91.

17. Sugimura H, Spratt EH, Compeau CG, et al. Thoracoscopic sympathetic clipping for hyperhidrosis: long-term results and reversibility. J Thorac Cardiovasc Surg 2009; 137:1370-6.

18. Currie AC, Evans JR, Thomas PR. An analysis of the natural course of compensatory sweating following thoracoscopic sympathectomy. Int J Surg 2011;9:437-9.

19. Bryant AS, Cerfolio RJ. Satisfaction and compensatory hyperhidrosis rates 5 years and longer after video-assisted thoracoscopic sympathotomy for hyperhidrosis. J Thorac Cardiovasc Surg 2014;147:1160-3.e1.

20. Vannucci F, Araujo JA. Thoracic sympathectomy for hyperhidrosis : from surgical indications to clinical results. J Thorac Dis 2017;9:S178-92.

21. Migliore M, Palazzolo M, Pennisi M, et al. Extended uniportal bilateral sympathectomy. J Vis Surg 2018;4:27.

22. Abu-Daff S, Maziak DE, Alshehab D, et al. Intrapleural fibrinolytic therapy (IPFT) in loculated pleural effusions analysis of predictors for failure of therapy and bleeding: a cohort study. BMJ Open 2013;3.
23. Scarci M, Abah U, Solli P, et al. EACTS expert consensus statement for surgical management of pleural empyema. Eur J Cardiothorac Surg 2015;48:642-53.

24. Chambers A, Routledge T, Dunning J, Scarci M. Is video-assisted thoracoscopic surgical decortication superior to open surgery in the management of adults with primary empyema? Interact Cardiovasc Thorac Surg 2010;11:171-7.

25. Bongiolatti S, Voltolini L, Borgianni S, et al. Uniportal thoracoscopic decortication for pleural empyema and the role of ultrasonographic preoperative staging. Interact Cardiovasc Thorac Surg 2017;24:560-6.

26. Rocco G, Martucci N, La Manna C, et al. Ten-year experience on 644 patients undergoing single-port (uniportal) video-assisted thoracoscopic surgery. Ann Thorac Surg 2013;96:434-8.

27. Rocco G, La Rocca A, La Manna C, et al. Uniportal video-assisted thoracoscopic surgery pericardial window. J Thorac Cardiovasc Surg 2006;131:921-2.

28. Bary MA, Abdel-aal KM, Mohamed RG, et al. Videoassisted thoracoscopic pericardial window for massive pericardial effusion: South Egypt experience. J Egypt Soc Cardio-Thoracic Surg 2017;25:73-8.

29. Trujillo-Reyes JC, Rami-Porta R, Caja SC, et al. Subxiphoid video-pericardioscopy. Multimed Man Cardiothorac Surg 2015;2015.

30. Rocco G. Single-Port Video-Assisted Thoracic Surgery (Uniportal) in the Routine General Thoracic Surgical Practice. Oper Tech Thorac Cardiovasc Surg 2009; 14:326-35.

31. Fabbrucci P, Nocentini L, Secci S, et al. Video-assisted thoracoscopy in the early diagnosis and management of post-traumatic pneumothorax and hemothorax. Surg Endosc 2008;22:1227-31.

32. Swierzy M, Faber S, Nachira D, et al. Uniportal videoassisted thoracoscopic surgery for the treatment of thoracic emergencies. J Thorac Dis 2018;10:S3720-5.

33. Wolfe GI, Kaminski HJ, Aban IB, et al. Randomized Trial of Thymectomy in Myasthenia Gravis. N Engl J Med 2016;375:511-22.

34. Rea F, Marulli G, Girardi R, et al. Long-term survival and prognostic factors in thymic epithelial tumours. Eur J Cardiothorac Surg 2004;26:412-8.

35. Regnard JF, Magdeleinat P, Dromer C, et al. Prognostic factors and long-term results after thymoma resection: a series of 307 patients. J Thorac Cardiovasc Surg 1996;112:376-84.

36. Hess NR, Sarkaria IS, Pennathur A, et al. Minimally 
invasive versus open thymectomy: a systematic review of surgical techniques, patient demographics, and perioperative outcomes. Ann Cardiothorac Surg 2016;5:1-9.

37. Friedant AJ, Handorf E, Su S, et al. Minimally invasive versus open thymectomy for thymic malignancies: systematic review and meta-analysis. J Thorac Oncol 2016;11:30-8.

38. Yang Y, Dong J, Y Huang. Thoracoscopic thymectomy versus open thymectomy for the treatment of thymoma: a meta-analysis. Eur J Surg Oncol 2016;42:1720-8.

39. Scarci M, Pardolesi A, Solli P. Uniportal video-assisted thoracic surgery thymectomy. Ann Cardiothorac Surg 2015;4:567-70.

40. Bedetti B, Solli P, Lawrence D, et al. Single port videoassisted thoracoscopic thymectomy. J Vis Surg 2016;2:149.

Cite this article as: Yeung C, Dawson J, Gilbert S. Uniportal video-assisted thoracoscopy approach to the management of non-pulmonary diseases of the chest. J Thorac Dis 2019;11(Suppl 16):S2062-S2068. doi: 10.21037/jtd.2019.03.21
41. Migliore M, Criscione A, Nardini M, et al. Single incision extended video assisted transcervical thymectomy. J Vis Surg 2017;3:154.

42. Caronia FP, Fiorelli A, Arrigo E, et al. Bilateral singleport thoracoscopic extended thymectomy for management of thymoma and myasthenia gravis: case report. J Cardiothorac Surg 2016;11:153.

43. Harris CG, James R, Tian D, et al. Systematic review and meta-analysis of uniportal versus multiportal videoassisted thoracoscopic lobectomy for lung cancer. Ann Cardiothorac Surg 2016;5:76-84.

44. Bertolaccini L, Viti A, Terzi A, et al. Geometric and ergonomic characteristics of the uniportal video-assisted thoracoscopic surgery (VATS) approach. Ann Cardiothorac Surg 2016;5:118-22. 